\title{
Study of the Knowledge Attitudes and Practices of the Dispensing of Anxiolytics in Pharmacies in Senegal
}

\author{
Jean Augustin Diegane Tine ${ }^{1, ~ *, ~ E r v e a n c e ~ B a n d i a k y ~}{ }^{1}$, Khadim Niang ${ }^{2}$, Oumar Bassoum ${ }^{1}$, \\ Adama Faye ${ }^{1}$, Ibrahima Seck ${ }^{1}$ \\ ${ }^{1}$ Faculty of Medicine, Pharmacy and Dentistry (FMPO), University Cheikh Anta Diop of Dakar (UCAD), Dakar, Senegal \\ ${ }^{2}$ Health Unit, University Gaston Berger (UGB) of Saint Louis, Dakar, Senegal
}

Email address:

leki105@yahoo.fr (J. A. D. Tine), jeanaugustindiegane.tine@ucad.edu.sn (J. A. D. Tine)

${ }^{*}$ Corresponding author

\section{To cite this article:}

Jean Augustin Diegane Tine, Erveance Bandiaky, Khadim Niang, Oumar Bassoum, Adama Faye, Ibrahima Seck. Study of the Knowledge Attitudes and Practices of the Dispensing of Anxiolytics in Pharmacies in Senegal. World Journal of Public Health.

Vol. 6, No. 2, 2021, pp. 52-58. doi: 10.11648/j.wjph.20210602.15

Received: May 19, 2021; Accepted: June 15, 2021; Published: June 22, 2021

\begin{abstract}
INTRODUCTION: An anxiolytic is a psychotropic drug used in the treatment of pathological anxiety. Because of the risks associated with the irrational use of these drugs, their prescription and dispensing must be strictly controlled. The objective of this research is to evaluate the knowledge, attitudes and practices of dispensing anxiolytics by providers of dispensing pharmacies in the region of Thies in Senegal. METHODOLOGY: This is an observational, cross-sectional, descriptive and analytical study at the level of providers of dispensing pharmacies in the departments of Mbour and Thies, in October 2018. Data collection was done by direct interview with administration of a questionnaire after informed consent from the provider. They were entered and analysed using EPI-Info. 7 software. RESULTS: A total of 221 pharmacy providers were surveyed. The average age of the providers was 36.8 years with a standard deviation of 9.5 years. Married people represented $68.8 \%$. Full time pharmacists represented $24.4 \%$. Anxiolytic dispensing practice was good in $78.3 \%$ of the providers. Good dispensing practice depended on professional level in the pharmacy (OR=14.0 (6.2 - 33.2)), good knowledge of pharmacological effects $(\mathrm{OR}=5.5(2.5-12.2))$ and good knowledge of adverse effects of anxiolytics $(\mathrm{OR}=5.0(2.0-12.2))$. The better the knowledge of anxiolytics, the better the dispensing practice. CONCLUSION: In order to improve the practice of dispensing anxiolytics and other psychotropic drugs in general, it will be necessary to strengthen the knowledge of providers through regular continuous training and to strengthen the supervision of dispensing sites by Ministry of Health inspectors.
\end{abstract}

Keywords: Dispensing, Anxiolytic, Pharmacist, Dispensaries, Senegal

\section{Introduction}

Subjected to acute stress and anxiety, people use anxiolytics. Their chronic use can lead to dependence and side effects on the liver, the nervous system and the respiratory system, as well as daytime hypovigilance which favours the occurrence of road accidents [1,2]. This is why the legislator places particular emphasis on those medicines containing so-called poisonous substances which have been classified in different tables or lists. Pharmaceutical legislation requires that these specific medicines be dispensed on the basis of a correctly written medical prescription, the conformity of which must first be certified by the pharmacist or his collaborators. [3]

The pharmacist is a health professional who can advise and inform the patient about medicines and health products. He is bound by pharmaceutical legislation and a code of ethics with many obligations to respect. Book V of the French Public Health Code relating to pharmacy is to a large extent applied in Senegal, even if for certain provisions legal instruments have been taken to readapt French law to the Senegalese national context. The field of medicine and pharmacy is one of the most regulated areas with numerous obligations to be respected. Indeed, medicines are not just another product; they are, among other things, a health good. This is why the manufacture, distribution, prescription and dispensing of medicines are highly regulated and must be strictly controlled 
to ensure compliance with the relevant laws and regulations and to apply sanctions if necessary. Due to an insufficient number of inspectors, the necessary control of psychotropic medicines is not always carried out properly. [3, 4]

Legislation thus plays an important role in the drug regulation process. In Senegal, the pharmacist is the only person authorised to prepare and dispense medicines and this monopoly is accompanied by an obligation to exercise the profession personally. Currently, the practice of pharmacists in pharmacies is not irreproachable and the control of pharmacies by public health inspectors does not always allow for a remedy. Due to their special nature, it is imperative that anxiolytics are not treated as simple medicines. Indeed, due to their financial accessibility, this therapeutic class is often used for other purposes such as drug addiction, a harmful and irrational use. Faced with the non-respect of pharmaceutical legislation concerning these drugs, such as the free sale of anxiolytics, self-medication, and the sale on advice by pharmacists, we deemed it necessary to study the knowledge, attitudes and practices of dispensing anxiolytics by pharmacists. In Senegal, few studies have been conducted on the dispensing of anxiolytics in pharmacies. [5]

The objective of this research is to evaluate the knowledge, attitudes and practices of the prescription of anxiolytics by pharmacists in pharmacies in the region of Thies in Senegal.

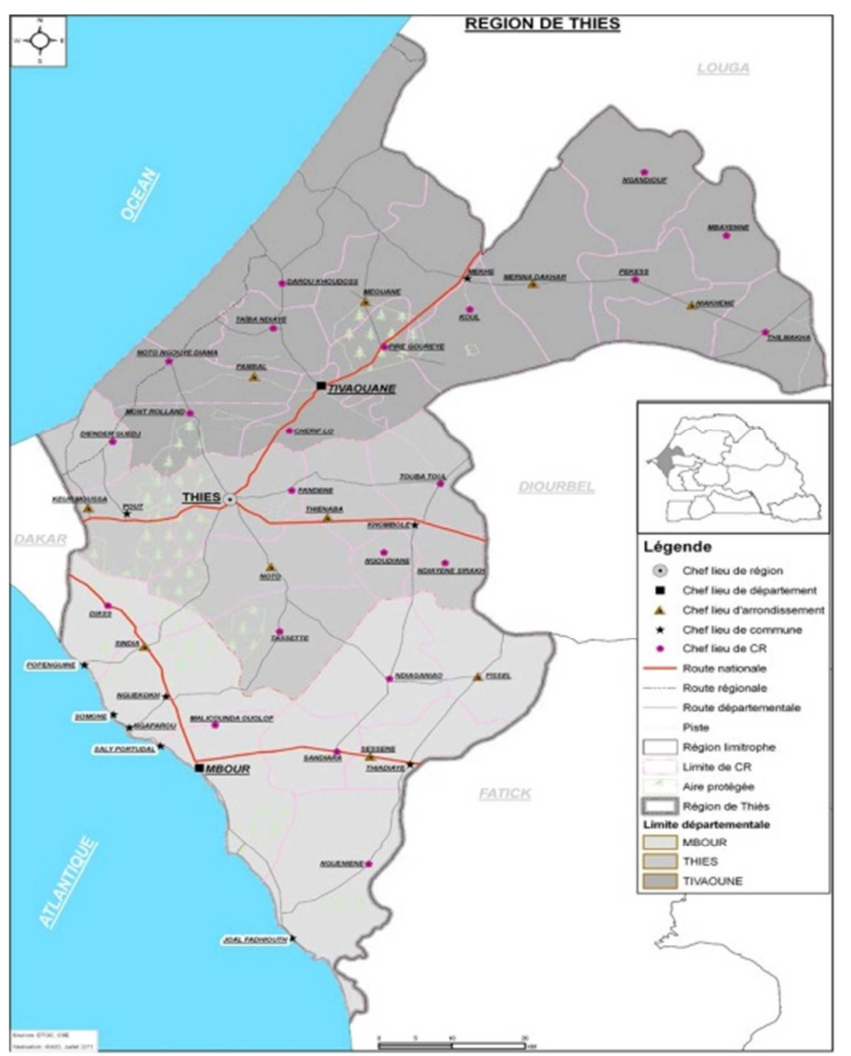

Figure 1. Map of the Thies region in Senegal (ANSD, 2018).

\section{Study Field}

Located $70 \mathrm{~km}$ from Dakar, the region of Thies is one of the 14 administrative regions of Senegal. It is located in the west of the country, around the Cape Verde peninsula. It covers an area of $6,601 \mathrm{~km}^{2}$, i.e. $3.4 \%$ of the national territory and is bordered to the north by the Louga region, to the south by the Fatick region, to the east by the Diourbel and Fatick regions and to the west by the Dakar region and the Atlantic Ocean.

The Thies region has three departments administered by departmental councils: Mbour, Thies and Tivaouane. The study framework is all the pharmacies in the departments of Mbour and Thies. [6]

The study framework is all pharmacies in the departments of Mbour and Thies. Out of a total of 1,100 pharmacies in Senegal up to 2018, the departments of Mbour and Thies have $11.7 \%$ of pharmacies.

\section{Methodology}

\subsection{Type of Study and Population}

This was an observational, cross-sectional and descriptive study among dispensing pharmacists in the departments of Mbour and Thies in October 2018.

We carried out an exhaustive recruitment which consisted of surveying all providers in pharmacies in the departments of Mbour and Thies.

\subsection{Data Collection}

Data were collected using a questionnaire that provided information on the sociodemographic characteristics of pharmacy providers, availability of anxiolytics, knowledge, attitudes and practices of providers on dispensing anxiolytics. The data was collected by direct interview with the administration of the questionnaire. It was carried out by five interviewers trained in the principles of anxiolytic prescription.

\subsection{Data Management and Analysis}

Data entry and analysis was carried out with EPI-Info. 7 statistical software. The description of qualitative variables was done with absolute and relative frequencies and that of quantitative variables with the mean and standard deviation.

The Student's t test and the ANOVA were used to make comparisons of means. For cases where the distribution was not normal, the non-parametric Wilcoxon test was used. For comparisons of proportions, the chisq 2 or Fischer test was used depending on the conditions of application. The risk was measured with the odds ratio (OR) with $95 \%$ confidence.

\subsection{Ethical Aspects}

Participation was free and informed. An information sheet was distributed to the respondents and their consent was recorded on a sheet before the start of each interview. Anonymity and confidentiality of information were respected during data processing. 


\section{Results}

\subsection{Description of Pharmacy and Provider Characteristics}

A total of 72 pharmacies agreed to participate in the study out of the 88 pharmacies visited (i.e. a participation rate of $82 \%$ ). Among the pharmacies that participated, 42 were in the department of Mbour out of a total of 55 pharmacies (i.e. $72.8 \%$ ) and 30 were in Thies out of a total of 42 pharmacies (71.4\%). The number of providers surveyed was 221, including 148 in Mbour and 73 in Thies. The main reasons for refusal were the absence of the pharmacist or lack of time on the part of the providers.

The average age of the providers was 36.8 years with a standard deviation of 9.5 years and a median of 35 years. The minimum age was 18 years and the maximum 58 years.

The average number of years of service of pharmacy providers was 9 years with a standard deviation of 7.1 years and a median of 7 years. The minimum number of years of experience was 1 year and the maximum 30 years. Table 1 gives the distribution of providers according to their sociodemographic characteristics.

Table 1. Distribution of pharmacy providers according to their socio-demographic characteristics.

\begin{tabular}{llll}
\hline Sociodemographic characteristics & Absolute frequencies (n) & Relative frequencies (\%) & Confidence interval (95\%) \\
\hline $\begin{array}{l}\text { Gender } \\
\text { Male }\end{array}$ & 128 & 57.9 & {$[51.1-64.5]$} \\
Female & 93 & 42.1 & \\
$\begin{array}{l}\text { Marital status } \\
\text { Unmarried }\end{array}$ & 67 & 30.3 & {$[35.5-48.9]$} \\
Married & 154 & 69,7 & {$[24.3-36.8]$} \\
$\begin{array}{l}\text { Religion } \\
\text { Muslim }\end{array}$ & 185 & 83.7 & {$[62,3-78,0]$} \\
Christian & 36 & 16.3 & {$[78.2-88.3]$} \\
$\begin{array}{l}\text { Profession } \\
\text { Senior pharmacists }\end{array}$ & 54 & 24.4 \\
$\begin{array}{l}\text { Junior pharmacists } \\
\text { Assistants in Pharmacy }\end{array}$ & 42 & 19 \\
\hline
\end{tabular}

\subsection{Pharmacy Providers' Knowledge of Anxiolytics}

The distribution of providers according to their knowledge of the pharmacological effects of anxiolytics showed that $81 \%$ of the providers knew about the sedative effect and $40.3 \%$ about the anticonvulsant effect. Moreover, $91.4 \%$ of the providers knew the pharmacological effects of anxiolytics. The study showed that half of the providers had a good level of knowledge of the pharmacological effects of anxiolytics while the other half had an average level of knowledge.

The duration of use of anxiolytics was known by $66.5 \%$ of providers out of a total of 221 respondents. The rest of the study population had no idea about the normal duration of use.

The side effects were less known by the providers. They were agitation (29.9\%); aggressiveness (24.4\%); irritability (23.5\%); anterograde amnesia (22.6\%); drug tolerance (20.4\%) and finally dependence known by $19.9 \%$ of the providers.

Of the 221 providers surveyed, $43.9 \%$ had a low level of knowledge of adverse effects. Table 2 shows the providers' knowledge of anxiolytics.

\subsection{Availability of Anxiolytics in Pharmacies}

The availability of anxiolytics varied according to the pharmacies surveyed. There were 25 pharmacies with full availability, 15 of which were located in the department of Mbour. There were 39 pharmacies with an availability between 60 and $90 \%, 22$ of which were in Mbour. For availability between $30-50 \%$, we had 8 pharmacies, 5 of which were in Mbour.

Among the anxiolytics available in the pharmacies surveyed, Alprazolam was in first place with $100 \%$ availability, followed by Bromazepam with $88.9 \%$ availability, then Diazepam with $83.3 \%$. Hydroxyzine and Clonazepam had availability of $72.2 \%$ and $70.8 \%$ respectively. For the rest of the anxiolytics found in pharmacies we obtained the following availability: Clonazepate (65.3\%), Prazepam (48.6\%), Tetrazepam (45.8\%), Zolpidem (44.4\%) and Oxazepam (36.1\%).

\subsection{Pharmacy Providers' Attitudes Towards Dispensing Anxiolytics}

The verification of the authenticity of an anxiolytic prescription by the provider must cover each element of the prescription. The providers systematically checked the prescriber's stamp $(94.1 \%)$; the patient's name $(82.8 \%)$; the prescriber's name $(81.4 \%)$; the dosage and date of prescription $(80.1 \%)$; the duration of use $(67.4 \%)$; the prescriber's address $(51.1 \%)$ and the prescriber's contact ( $42.5 \%$ of the providers). The study showed that $53.9 \%$ of the respondents had a good level of analysis of an anxiolytic prescription while $46.1 \%$ of the providers had an average level. The study showed that $50.2 \%$ of the providers gave advice on the risks related to the irrational use of anxiolytics only when needed, $29 \%$ on the client's request and $25.3 \%$ of the providers declared giving advice at each client's visit to the pharmacy.

The study showed that $24.9 \%$ of the providers surveyed agreed to dispense anxiolytics upon spontaneous request while $75.1 \%$ systematically refused to dispense. Table 3 shows the distribution of respondents according to their attitude towards dispensing anxiolytics. 
Table 2. Distribution of pharmacy providers according to knowledge of anxiolytics.

\begin{tabular}{|c|c|c|c|}
\hline Knowledge Items & Absolute frequencies (n) & Relative frequencies $(\%)$ & Confidence intervals $(\mathbf{9 5 \%})$ \\
\hline \multicolumn{4}{|c|}{ Pharmacological effects of anxiolytics } \\
\hline \multicolumn{4}{|c|}{ Sedative } \\
\hline Yes & 179 & 81.0 & {$[75.2-85.9)$} \\
\hline No & 42 & 19.0 & {$[14.1-24.8]$} \\
\hline \multicolumn{4}{|l|}{ Anxiolytic } \\
\hline Yes & 138 & 62.4 & [31.2-44.3] \\
\hline No & 83 & 37.6 & {$[55.7-68.9]$} \\
\hline \multicolumn{4}{|l|}{ Anticonvulsant } \\
\hline Yes & 89 & 40.3 & [33.8-47.1] \\
\hline No & 132 & 59.7 & [52.9-66.3] \\
\hline \multicolumn{4}{|l|}{ Muscle relaxant } \\
\hline Yes & 131 & 59.3 & {$[52.5-65.8]$} \\
\hline No & 90 & 40.7 & {$[34.2-47.5]$} \\
\hline \multicolumn{4}{|c|}{ Side effects or risks of anxiolytics } \\
\hline \multicolumn{4}{|c|}{ Drowsiness } \\
\hline Yes & 83 & 82.8 & {$[77.2-87.5]$} \\
\hline No & 38 & 17.2 & {$[12.5-22.8]$} \\
\hline \multicolumn{4}{|l|}{ Behavioural disorder } \\
\hline Yes & 133 & 60.2 & {$[53.4-66.7]$} \\
\hline No & 88 & 39.8 & [33.3-46.6] \\
\hline \multicolumn{4}{|l|}{ Confusion } \\
\hline Yes & 97 & 43.9 & {$[37.2-50.7]$} \\
\hline No & 124 & 56.1 & {$[49.3-62.8]$} \\
\hline \multicolumn{4}{|l|}{ Libido disorders } \\
\hline Yes & 93 & 42.1 & [35.5-48.9] \\
\hline No & 128 & 57.9 & [51.1-64.5] \\
\hline \multicolumn{4}{|l|}{ Hypovigilance } \\
\hline Yes & 82 & 37.1 & {$[30.7-43.8]$} \\
\hline No & 139 & 62.9 & {$[56.2-69.3]$} \\
\hline \multicolumn{4}{|c|}{ Impaired psychomotor function } \\
\hline Yes & 70 & 31.7 & {$[25.6-38,3]$} \\
\hline No & 151 & 68.3 & {$[61.7-74.4]$} \\
\hline \multicolumn{4}{|l|}{ Agitation } \\
\hline Yes & 66 & 29.9 & {$[23.9-36.4]$} \\
\hline No & 155 & 70.1 & {$[63.6-76.1]$} \\
\hline \multicolumn{4}{|l|}{ Aggressiveness } \\
\hline Yes & 54 & 24.4 & [18.9-30.7] \\
\hline No & 167 & 75.6 & [69.4-81.1] \\
\hline \multicolumn{4}{|l|}{ Irritability } \\
\hline Yes & 52 & 23.5 & [18.1-29.7] \\
\hline No & 169 & 76.5 & [70.3-81.9] \\
\hline \multicolumn{4}{|c|}{ Anterograde amnesia } \\
\hline Yes & 50 & 22.6 & {$[17.3-28.7]$} \\
\hline & 171 & 77.4 & {$[71,3-82.7]$} \\
\hline \multicolumn{4}{|l|}{$\begin{array}{l}\text { No } \\
\text { Tolerance }\end{array}$} \\
\hline Yes & 45 & 20.4 & {$[15,3-26.3]$} \\
\hline No & 176 & 79.6 & {$[73.7-84.7]$} \\
\hline \multicolumn{4}{|l|}{ Addiction } \\
\hline Yes & 44 & 19.9 & [14.9-25.8] \\
\hline No & 177 & 80.1 & {$[74.2-85.2]$} \\
\hline Overall level of kno & effects & & \\
\hline Good & 113 & 51.1 & [44.3-57.9] \\
\hline Meduim & 108 & 48.9 & [42.1-55.7] \\
\hline Overall level of kno & & & \\
\hline Good & 53 & 24.0 & {$[18.5-30.2]$} \\
\hline Meduim & 71 & 32.1 & {$[26.0-38.7]$} \\
\hline Low & 97 & 43.9 & {$[37.2-50.7]$} \\
\hline
\end{tabular}

\subsection{Pharmacy Providers' Practices in Dispensing Anxiolytics}

Assistance in dispensing anxiolytics was noted in $50.7 \%$ of providers. Advice on anxiolytics was observed in $23.53 \%$ of the respondents.

The good practice of dispensing anxiolytics was noted in $78.7 \%$ of the respondents with a $95 \%$ confidence interval between 72.7 and 83.9. Table 4 shows the distribution of pharmacy providers' anxiolytic dispensing practices. 
Table 3. Distribution of pharmacy providers according to attitude towards an anxiolytic prescription.

\begin{tabular}{|c|c|c|c|}
\hline Pharmacists' attitudes & Absolute frequencies (n) & Relative frequencies $(\%)$ & Confidence intervals (95\%) \\
\hline \multicolumn{4}{|c|}{ Verification of elements of the authenticity of the prescription } \\
\hline \multicolumn{4}{|c|}{ Prescriber's stamp } \\
\hline Yes & 208 & 94.1 & [91.2-97.5] \\
\hline No & 13 & 5.9 & {$[2.5-8.8]$} \\
\hline \multicolumn{4}{|l|}{ Patient's name } \\
\hline Yes & 183 & 82.8 & {$[77.9-88.2]$} \\
\hline No & 38 & 17.2 & {$[11.8-22.0]$} \\
\hline \multicolumn{4}{|l|}{ Name of the prescriber } \\
\hline Yes & 180 & 81.4 & {$[76.5-87.0]$} \\
\hline No & 41 & 18.6 & {$[12.1-23.5]$} \\
\hline \multicolumn{4}{|l|}{ Dosage } \\
\hline Yes & 177 & 80.1 & {$[75.0-85.8]$} \\
\hline No & 44 & 19.9 & {$[14.2-25.0]$} \\
\hline \multicolumn{4}{|l|}{ Prescription date } \\
\hline Yes & 177 & 80.1 & [75.4-86.2] \\
\hline No & 44 & 19.9 & [13.9-24.6] \\
\hline \multicolumn{4}{|l|}{ Duration of use } \\
\hline Yes & 149 & 67.4 & {$[61.4-74.2]$} \\
\hline No & 72 & 32.6 & {$[25.8-38.6]$} \\
\hline \multicolumn{4}{|l|}{ Address of the prescriber } \\
\hline Yes & 113 & 51.1 & {$[44.7-58.4]$} \\
\hline No & 108 & 48.9 & [41.6-55.2] \\
\hline \multicolumn{4}{|l|}{ Prescriber's contact } \\
\hline Yes & 94 & 42.5 & {$[36.3-49.8)$} \\
\hline No & 127 & 57.5 & {$[50.2-63.7]$} \\
\hline \multicolumn{4}{|c|}{ Advising clients on the risks of misuse of anxiolytics } \\
\hline \multicolumn{4}{|c|}{ On request } \\
\hline Yes & 64 & 29.0 & [23.1-35.4] \\
\hline No & 157 & 71.0 & [64.6-76.9] \\
\hline \multicolumn{4}{|l|}{ At each visit } \\
\hline Yes & 56 & 25.3 & {$[19.7-31.6]$} \\
\hline No & 165 & 74.7 & [68.4-80.3] \\
\hline \multicolumn{4}{|l|}{ Only if necessary } \\
\hline Yes & 111 & 50.2 & {$[43.4-57.0]$} \\
\hline No & 110 & 49.8 & {$[43.0-56.6]$} \\
\hline \multicolumn{4}{|c|}{ Pharmacy provider's decision on a non-prescription request for anxiolytics } \\
\hline Acceptance of delivery & 55 & 24.9 & [16.9-28.3] \\
\hline Refusal to issue & 166 & 75.1 & {$[68.9-80.7]$} \\
\hline \multicolumn{4}{|c|}{ Level verification of the quality of the prescription } \\
\hline Good & 119 & 53.9 & {$[47.0-60.5]$} \\
\hline Medium & 102 & 46.1 & [39.5-53.0] \\
\hline
\end{tabular}

Table 4. Distribution of pharmacy providers according to their anti-anxiety dispensing practices.

\begin{tabular}{lccc}
\hline Pharmacy providers' pratices & Absolute frequencies (n) & Relative frequencies (\%) & Confidence intervals (95\%) \\
\hline Assistance in filling the prescription for anxiolytics & 112 & 50.7 & {$[44.1-57.7]$} \\
Yes & 109 & 49.3 & {$[42.3-55.9]$} \\
No & 52 & & {$[18.3-29.9]$} \\
Advice on the use of anti-anxiety drugs among pharmacy customers & 169 & 23.5 & {$[70.1-81.7]$} \\
Yes & 174 & 76.5 & {$[72.7-83.9]$} \\
No & 47 & 78.7 & {$[16.1-27.3]$} \\
Registration of the prescription in the pharmacy register & 21.3 & {$[38.6-52.1]$} \\
Yes & 100 & 45.3 & {$[47.9-61.4]$} \\
No & 121 & 54.7 & {$[72.7-83.9]$} \\
Continuous training on the dispensing of anxiolytics & & {$[16.1-27.3]$} \\
Yes & 174 & 78.3 & 21.3 \\
Quality of dispensing practice of anxiolytics & 47 & & \\
Good & &
\end{tabular}

\subsection{Analysis of Factors Associated with Good Dispensing Practice for Anxiolytics}

Pharmacists with tenure were more compliant with dispensing rules than others with an OR of 14.0 (6.2 - 33.2).

Good dispensing practice was most common among providers who had good knowledge of the pharmacological effects 
$(\mathrm{OR}=5.5(2.5-12.2))$ and adverse effects of anxiolytics $(\mathrm{OR}=5.0(2.0-12.2))$. Table 5 shows the ORs for factors associated with good practice in dispensing anxiolytics.

Table 5. Factors explaining the good practice of dispensing anxiolytics.

\begin{tabular}{lll}
\hline & \multicolumn{2}{c}{ Good practice in dispensing anxiolytics at pharmacies } \\
\hline & Odds ratio & $\mathbf{9 5 \%}$ CI \\
\hline Gender & 1 & - \\
Female & 1.5 & $(0.7-3.0)$ \\
Male & & - \\
Marital status & 1 & $(1.1-5.6)$ \\
Unmarried & 2.4 & - \\
Married & 1 & $(6.2-33.2)$ \\
Profession & 14.0 & $(0.8-6.4)$ \\
Assistants in Pharmacy & 2.3 & \\
Senior pharmacists & 1 & - \\
Junior pharmacists & 5.5 & $(2.5-12.2)$ \\
Overall level of knowledge of the pharmacological effects of anxiolytics & - \\
Medium & 1 & $(1.6-9.0)$ \\
Good & 3.8 & $(2.0-12.2)$ \\
Overall level of knowledge of the adverse effects of anxiolytics & \\
Low & 1.0 & \\
Medium & Good &
\end{tabular}

\section{Discussion}

The profession of pharmacist in Senegal is dominated by men. This study found a male predominance of $57 \%$ against $42.1 \%$ for women, with a sex ratio of 1.4 . These results are similar to those of the study conducted in pharmacies in Mali with a male predominance of $72.7 \%$ and in Burkina Faso with $57.9 \%$ [7]. The average age of the respondents was about 37 years with an average length of service in the pharmacy of 9 years. Pharmacy assistants were in the majority at $56.6 \%$. This difference is explained by the fact that pharmacists were often absent from the pharmacies surveyed. Assistants made more sales than the incumbents and second-line pharmacists in most of the pharmacies.

This study found that the most commonly dispensed anxiolytics in these pharmacies were Diazepam, Alprazolam, Bromazepam, Hydroxyzine and Clonazepam.

Dispensing anxiolytics by advice was observed in $23.5 \%$ of providers. Another study done in Dakar on the knowledge, attitudes and practices of pharmacy providers on drug dispensing in 2013 showed a dispensation of $8.3 \%$ by advice [8], while in Tanzania it was $32 \%$ in private pharmacies [9]. In Senegal, there has been an increase in the demand for medical consultation in pharmacies in the form of ad hoc advice. This practice is rapidly becoming the first recourse for primary health care services. In Mbour, tourists who are used to the use of anxiolytics resort to pharmacists to renew their prescriptions or to buy them directly.

Good practice in dispensing anxiolytics was noted in $78.7 \%$ of pharmacy providers. In Ethiopia, a study on the dispensing of emergency contraception by pharmacy providers showed a frequency of $85 \%$ of good dispensing practice [10]. A study in Abidjan showed $14.1 \%$ good dispensing practice for antibiotics at the pharmacy level [11].

Pharmacist intervention during the dispensing of an anxiolytic prescription was noted in $50.7 \%$ of cases and transcription of the prescription on the prescription pad was noted in $78.1 \%$ of cases.

Half of the providers were aware of the pharmacological effects of anxiolytics (51.1\%), but their adverse effects were not well known by these providers $(24 \%)$.

Pharmacists had a better practice of dispensing anxiolytics with an $\mathrm{OR}=14.0$ (6.2 - 33.2). The higher the level of education, the better the dispensing. Unfortunately, we found that assistants did more dispensing in pharmacies. Good knowledge of the pharmacological effects $(\mathrm{OR}=5.5(2.5-$ 12.2)) and side effects of anxiolytics $(\mathrm{OR}=5.0(2.0-12.2))$ is decisive for good dispensing practice. The better the knowledge, the better the dispensing of anxiolytics.

The main limitation of this study is the lack of direct observation of anxiolytic dispensing practice in some pharmacies due to the lack of consent of the pharmacy manager. The main reasons given by the pharmacy managers were lack of time and respect for the privacy of the customer.

\section{Conclusion}

Excessive consumption of anxiolytic drugs leads to psychological disturbances and serious effects on the liver, the nervous system and the respiratory system. Daytime hypovigilance, which favours the occurrence of accidents, particularly at work, at home and on the road. This is why pharmaceutical legislation requires that these specific medicines be dispensed on the basis of a correctly written medical prescription, the conformity of which must be certified beforehand by the pharmacist or his staff.

The pharmacist must advise and inform the patient about medicines and health products. He is obliged to respect a code of ethics based on ethics and professionalism. The level of analysis of the anxiolytic prescription was correct with $53.9 \%$ and $75 \%$ of the providers had refused to dispense 
anxiolytics upon spontaneous request. The proportion of providers who had good practice in dispensing anxiolytics was $78.3 \%$. The factors explaining this good practice were the level of professionalism and the good knowledge of the pharmacological and undesirable effects of anxiolytics. It is recommended that the training of pharmacy providers in the dispensing of anxiolytics and more generally of psychotropic drugs be reinforced.

\section{References}

[1] GERARD A. On the proper use of psychotropic drugs. The doctor, the patient and the drugs. Paris: Albin Michel; 2005. $212 \mathrm{p}$.

[2] DUROY D, LEJOYEUX M. Addiction to psychotropic drugs (benzodiazepines and related) [ECN Item 75]. Rev Prat Monogr. Nov 2016; (Tome 66, nº): e395-8.

[3] LAGET J. Prescription of psychotropic drugs in adolescents. 2000. $117-122 \mathrm{p}$.

[4] GRANGER B, JALFRE V. Les psychotropes: idées reçues [Internet]. Paris: Le Cavalier Bleu; 2010. 128 p. (Idées reçues) Disponible sur: http://www.amazon.fr/psycotropes-BernardGranger/dp/2846703256/ref=sr_1_1?ie=UTF8\&qid=1292939 $152 \& \mathrm{sr}=1-1$.

[5] Organization WH. WHO pharmaceutical strategy: framework for action on essential medicines and pharmaceutical policies, 2000-2003. 2000 [cited 18 May 2021]; Available from: https://apps.who.int/iris/handle/10665/66934.
[6] ANSD. Map of the Thies region [Internet]. Agence Nationale de la Statistique et de la Démographie. 2018 [cited 18 May 2021]. Available from: htps://www.ansd.sn/index.php?option=com_regions\&view=re gion\&id $=5$.

[7] Ganfon H, Diallo T, Nanga C, Coulibaly N, Benao V, Ekanmian $\mathrm{G}$, et al. Knowledge and practices of malaria management by private pharmacy staff in five major cities of Benin, Burkina Faso and Mali in 2014. Med Sante Tropicales [Internet]. Med Sante Trop. 2017 [cited 18 May 2021]; 164-9. Available from: https://bec.uac.bj/uploads/publication/84165b6755ea6c527f3983d 539df67ae.pdf.

[8] Sene M, Ndiaye C, Diouf I, Kebe A, Diaw M, Ba A, et al. Emergency contraception: levels of knowledge, attitudes and risks related to its use in a population of women in the Dakar region. J Sci Technol [Internet]. 2013 [cited 18 May 2021]; 11 (1): 35-44. Available from: http://jst.ucad.sn/images/stories/articles/article\%20Sene.pdf.

[9] Kagashe GAB, Minzi O, Matowe L. An assessment of dispensing practices in private pharmacies in Dar-es-Salaam, Tanzania. Int J Pharm Pract. Feb 2011; 19 (1): 30-5.

[10] Belachew SA, Yimenu DK, Gebresillassie BM. Pharmacy Professionals' Dispensing Practice, Knowledge, and Attitude towards Emergency Contraceptives in Gondar Town, Northwestern Ethiopia: A Cross-Sectional Study. Int J Reprod Med [Internet]. 10 Jul 2017 [cited 18 May 2021]; 2017: e8754126. Available from: https://www.hindawi.com/journals/ijrmed/2017/8754126/.

[11] Hounsa A, De Mol P. [Antibiotics dispensed upon the recommendation of staff in private dispensaries in Abidjan in the Ivory Coast]. J Pharm Belg. Sept 2009; (3): 99-104. 